\title{
CHARACTERIZING THE COOL KOIs. VII. REFINED PHYSICAL PROPERTIES OF THE TRANSITING BROWN DWARF LHS 6343 C
}

\author{
Benjamin T. Montet ${ }^{1,2}$, John Asher Johnson ${ }^{2}$, Philip S. Muirhead ${ }^{3}$, Ashle Villar ${ }^{2}$, Corinne Vassallo $^{4}$, \\ Christoph Baranec ${ }^{5}$, Nicholas M. LaW ${ }^{6}$, Reed Riddle ${ }^{1}$, Geoffrey W. Marcy ${ }^{7}$, \\ ANDREW W. HOWARD ${ }^{8}$, AND HOWARD IsAaCSON ${ }^{7}$ \\ ${ }^{1}$ Cahill Center for Astronomy and Astrophysics, California Institute of Technology, 1200 E. California Blvd., \\ MC 249-17, Pasadena, CA 91106, USA; btm@astro.caltech.edu \\ ${ }^{2}$ Harvard-Smithsonian Center for Astrophysics, 60 Garden Street, Cambridge, MA 02138, USA \\ ${ }^{3}$ Department of Astronomy, Boston University, 725 Commonwealth Avenue, Boston, MA 02215, USA \\ ${ }^{4}$ Department of Aerospace Engineering and Engineering Mechanics, The University of Texas at Austin, 210 East 24th Street, Austin, TX 78712, USA \\ ${ }^{5}$ Institute for Astronomy, University of Hawaii at Mānoa, Hilo, HI 96720, USA \\ ${ }^{6}$ Department of Physics and Astronomy, University of North Carolina at Chapel Hill, Chapel Hill, NC 27599, USA \\ ${ }^{7}$ Department of Astronomy, University of California, Berkeley, CA 94720, USA \\ ${ }^{8}$ Institute for Astronomy, University of Hawaii, 2680 Woodlawn Drive, Honolulu, HI 96822, USA \\ Received 2014 November 12; accepted 2014 December 28; published 2015 February 20
}

\begin{abstract}
We present an updated analysis of LHS 6343, a triple system in the Kepler field which consists of a brown dwarf transiting one member of a widely separated M+M binary system. By analyzing the full Kepler data set and $34 \mathrm{Keck} /$ HIgh Resolution Echelle Spectrometer radial velocity observations, we measure both the observed transit depth and Doppler semiamplitude to $0.5 \%$ precision. With Robo-AO and Palomar/PHARO adaptive optics imaging as well as TripleSpec spectroscopy, we measure a model-dependent mass for LHS 6343 C of $62.1 \pm 1.2 M_{\text {Jup }}$ and a radius of $0.783 \pm 0.011 R_{\text {Jup }}$. We detect the secondary eclipse in the Kepler data at $3.5 \sigma$, measuring $e \cos \omega=0.0228 \pm 0.0008$. We also derive a method to measure the mass and radius of a star and transiting companion directly, without any direct reliance on stellar models. The mass and radius of both objects depend only on the orbital period, stellar density, reduced semimajor axis, Doppler semiamplitude, eccentricity, and inclination, as well as the knowledge that the primary star falls on the main sequence. With this method, we calculate a mass and radius for LHS $6343 \mathrm{C}$ to a precision of $3 \%$ and $2 \%$, respectively.
\end{abstract}

Key words: binaries: general - brown dwarfs - stars: fundamental parameters - stars: individual (KIC 10002261) stars: late-type - stars: low-mass

\section{INTRODUCTION}

The growth of brown dwarf astronomy has closely mirrored that of exoplanetary astronomy. Although Latham et al. (1989) discovered a likely brown dwarf candidate, the first confirmed detection of a brown dwarf was announced two months before the announcement of the first exoplanet orbiting a mainsequence star (Rebolo et al. 1995; Mayor \& Queloz 1995). That same year also saw the discovery of the first brown dwarf orbiting a stellar-mass companion (Nakajima et al. 1995). Today, more than 2000 brown dwarfs have been discovered. The majority of these substellar objects have no detected companions, so characterization is often limited to spectroscopic observations. In these cases, the atmosphere of the brown dwarf can be extensively studied (e.g., Burgasser et al. 2014; Faherty et al. 2014), but its physical parameters, including mass and radius, cannot be measured directly.

When a brown dwarf with a gravitationally bound companion is detected, detailed characterization of its physical properties is possible. Radial velocity (RV) surveys have produced a significant number of brown dwarf candidates with minimum mass determinations (e.g., Patel et al. 2007). Astrometric monitoring of directly imaged brown dwarf companions to stars has led to dynamical mass measurements of brown dwarfs (Liu et al. 2002; Dupuy et al. 2009; Crepp et al. 2012). While there are many brown dwarfs with measured masses, radii can only be directly measured in transiting or eclipsing systems. The first eclipsing brown dwarf system, discovered by Stassun et al. (2006) in the Orion Nebula, produced the first measurement of a brown dwarf's radius and the first test of theoretical mass-radius relations. Today, there are 11 brown dwarfs with measured masses and radii (Díaz et al. 2014). Of this sample, eight transit a stellarmass companion and only four are not inflated due to youth or irradiation. If the brown dwarf is assumed to be coeval with its host star, the brown dwarf's age and metallicity can be estimated. Both properties are expected to affect the brown dwarf mass-radius relation, making observations of transiting brown dwarfs especially valuable (Burrows et al. 2011).

Recently, four brown dwarfs have been detected by the Kepler mission (Johnson et al. 2011; Bouchy et al. 2011a; Díaz et al. 2013; Moutou et al. 2013). Launched in 2009, the Kepler telescope collected wide-field photometric observations of approximately 200,000 stars in Cygnus and Lyra every 30 minutes for $4 \mathrm{yr}$ (Borucki et al. 2010). The mission was designed as a search for transiting planets. As brown dwarfs have radii similar to Jupiter, brown dwarfs were also easily detected; only a few $\mathrm{RV}$ observations are necessary to distinguish between a giant planet and brown dwarf companion (e.g., Moutou et al. 2013).

The first unambiguous brown dwarf detected from Kepler data was found in the LHS 6343 system and announced by Johnson et al. (2011, hereafter J11). The authors analyzed five transits of the primary star observed in the first six weeks of Kepler data, combined with one transit observed in the $Z$ band with the Nickel Telescope at Lick Observatory and $14 \mathrm{RV}$ observations with Keck/HIgh Resolution Echelle Spectrometer (HIRES). The authors also obtained PHARO adaptive optics imaging data from the Palomar 200 inch telescope, imaging a companion $0.5 \mathrm{mag}$ fainter than the primary at a separation of $0 ! 7$. From these 
observations, the authors were able to measure a mass for the brown dwarf of $62.7 \pm 2.4 M_{\text {Jup }}$, a radius of $0.833 \pm 0.021 R_{\text {Jup }}$, and a period of 12.71 days, corresponding to a semimajor axis of $0.0804 \pm 0.0006 \mathrm{AU}$. The authors define LHS $6343 \mathrm{~A}$ as the primary star, LHS $6343 \mathrm{~B}$ as the widely separated binary M dwarf, and LHS $6343 \mathrm{C}$ as the brown dwarf orbiting the A component, and note the architecture of this system is very similar to the NLTT 41135 system discovered by Irwin et al. (2010).

Additional papers have expanded our knowledge of LHS 6343. Southworth (2011) re-fit the Kepler light curve, using data through Quarter 2 from the mission. By fitting the observations using five different sets of stellar models, he attempted to reduce biases caused by any one individual stellar model. He found different models provide a consistent brown dwarf radius at the $0.08 R_{\mathrm{Jup}}$ level, but found a higher mass than $\mathrm{J} 11$ : his best-fitting mass for LHS $6343 \mathrm{C}$ was $70 \pm 6 M_{\text {Jup }}$ Oshagh et al. (2012) analyzed the lack of transit timing variations in the system, finding that any additional companions to LHS $6343 \mathrm{~A}$ with an orbital period smaller than 100 days must have a mass smaller than that of Jupiter. With six quarters of Kepler data, Herrero et al. (2013) measured a photometric rotation period of $13.13 \pm 0.02$ days for LHS $6343 \mathrm{~A}$. The authors also claimed to observe spot-crossing events during the transits of LHS 6343 A, as well as out-of-transit photometric modulation with a period consistent with the orbital period of LHS 6343 C. Herrero et al. (2014) updated this work, concluding that the out-of-transit variations are dominated by relativistic Doppler beaming.

In many of the papers about the LHS 6343 system after the discovery paper, the authors assumed the physical parameters of J11. This is not necessarily an ideal assumption to make. J11 used a limited data set during their analysis. Their photometry consisted of only 6 transits and $14 \mathrm{RVs}$, and they estimated the third light contribution of LHS 6343 B by extrapolating from near-infrared (IR) observations to the Kepler bandpass. Moreover, the derived stellar parameters in that paper were based only on photometric observations and depend strongly on the accuracy of the Padova model grids (Girardi et al. 2002) upon which they are based.

The conclusion of the primary Kepler mission affords us an opportunity to reanalyze the LHS 6343 system using the complete Kepler data set. Such a reanalysis enables us to better measure the brown dwarf's mass and radius. There are only three non-inflated brown dwarfs with both a mass and radius measured to $5 \%$ or better: LHS 6343 C, KOI-205 b (Díaz et al. 2013), and KOI-415 b (Moutou et al. 2013). To test theoretical brown dwarf evolutionary models, we would like to measure the masses, radii, and metallicities of these objects as precisely as possible. In this work, we analyze the full Kepler data set for this object to measure the transit profile. We combine this light curve with additional RV observations, near-IR spectroscopy of LHS $6343 \mathrm{AB}$, and Robo-AO visible-light adaptive optics. Without any reliance on stellar models beyond an empirical main-sequence mass-radius relation, we are able to measure the mass of LHS $6343 \mathrm{C}$ to a precision of $3 \%$ and the radius to a precision of $2 \%$. The mass and radius measurements depend only on the following parameters, all measured directly from the data: the orbital period, stellar density $\rho_{\star}$, reduced semimajor axis $a / R_{\star}$, Doppler semiamplitude $K$, eccentricity, and inclination. Our technique allows one to calculate the mass and radius for both members of a transiting system. We also combine our data with the predictions for the mass of LHS 6343 A from the Dartmouth stellar evolutionary models of Dotter et al. (2008). These combined data enable us to measure a model-dependent mass and radius of LHS $6343 \mathrm{C}$ to better than $2 \%$ each; we also measure a metallicity of the system of $0.02 \pm 0.19$ dex.

In Section 2 we describe the observations used in this paper. In Section 3 we outline our data analysis pipeline. In Section 4 we present our results. In Section 5 we summarize our present efforts and outline our future plans to measure the brown dwarf's luminosity. In the Appendix, we derive the relation between transit and RV parameters and the mass and radius of both the primary and secondary companion.

This study presents, to date, the most precise mass and radius measurements of a non-inflated brown dwarf. Observations such as these are essential for future detailed characterization of field brown dwarfs.

\section{OBSERVATIONS}

\subsection{Kepler Photometry}

The LHS 6343 system (KIC 10002261, KOI-959) was part of the initial Kepler target selection and was observed during all observing quarters in long cadence mode. Between 2011 February 22 and 2011 March 14, the system was also observed using Kepler's short cadence mode, with observations collected every $58.84876 \mathrm{~s}$ in the reference frame of the spacecraft. We downloaded the entire data set from the NASA Multimission Archive at STScI (MAST).

For both long and short cadence observations, Kepler data consist of a postage stamp containing tens of pixels, a small number of which are combined to form an effective aperture. The flux from all pixels in the aperture are combined to create a light curve. The Kepler team defines an aperture for all targets and performs aperture photometry as a part of their Photometric Analysis (PA) pipeline, which produces a light curve from the pixel-level data (Jenkins et al. 2010). This pipeline also removes the photometric background and cosmic rays.

In analyzing the pipeline-generated light curve, we detected occasional anomalies during transit events, with the recorded flux systematically larger than expected. These anomalies were also detected by Herrero et al. (2013), who attribute them to occultations of spots on LHS 6343 A by LHS 6343 C. The anomalies occur only in the long cadence data, and only when the transit is symmetric around one data point in the Kepler time series, so that the central in-transit flux measurement would be expected to be significantly lower than the surrounding data points. By investigating the pixel-level data, we find that each anomaly has been registered as a cosmic ray by the PA pipeline, and "corrected" to an artificially large value.

Using the pixel-level data, recorded before the cosmic ray correction in the pipeline, we removed these artificial corrections. We find the anomalies can be completely explained as false cosmic ray detections: there is no evidence for transit-to-transit variability in the Kepler data.

We expect stellar granulation to induce correlated photometric variability only at a level significantly below the precision of our observations. Correlated noise attributed to stellar granulation has been previously observed when modeling transits of companions to higher mass stars (e.g., Huber et al. 2013) and used to derive fundamental parameters of the stars themselves (Bastien et al. 2013). Both the timescale and magnitude of the correlated noise are inversely proportional to the stellar density (Gilliland et al. 2010). For an M dwarf with a mass around $0.3 M_{\odot}$, we expect granulation to induce correlated noise with a period of approximately $10 \mathrm{~s}$ and an amplitude of $50 \mathrm{ppm}$ 
(Winget et al. 1991). Therefore, given the precision and cadence of the Kepler observations we do not expect to observe correlated noise due to granulation in the LHS 6343 system.

We tested for correlated noise on transit timescales by calculating the autocorrelation matrix for out-of-transit sections of the data. For both long cadence and short cadence data, all offdiagonal elements have absolute values less than 0.03 ; we found no periodic structure to the autocorrelation matrix. Therefore, on transit timescales the noise can be treated as white.

We converted all times recorded by Kepler to barycentric dynamical time (TDB), not UTC, which was mistakenly recorded during the first $3 \mathrm{yr}$ of the mission. As a result, our times differ from those reported in the analysis of J11 by $66.184 \mathrm{~s}$.

We then detrended the light curve to remove the effects of stellar and instrumental variability. For all transit events with at least four data points recorded continuously before and after the transit, we selected a region bounded by a maximum of three transit durations on either side of the nominal transit center. If there is any spacecraft motion, such as a thruster fire or data downlink, we clipped the fitting region to not include these data. We then fit a second-order polynomial to the out-of-transit flux. We normalized the light curve by dividing the observed flux values by the calculated polynomial. We repeated this procedure near the midpoint between successive transits in order to search for evidence of a secondary eclipse. We estimated the noise level in the data by measuring the variance observed in the out-of-transit segments of the data.

\subsection{Keck/HIRES Radial Velocities}

We obtained spectroscopic observations of LHS 6343 using the HIRES $(R \approx 48,000)$ at the W. M. Keck Observatory. All observations were taken using the $\mathrm{C} 2$ decker. With a projected length of 14 ". 0 , the decker enables accurate sky subtraction. The first four observations were obtained using a 45 minute exposure time and the standard iodine cell setup described by Howard et al. (2010). Once LHS 6343 C was identified as an transiting brown dwarf, the remaining observations were obtained with 3 minute exposure times and without the iodine cell. For all observations, the slit was aligned along the binary axis so that light from both stars fell upon the detector.

To measure the RV of LHS 6343 A, we used LHS 6343 B as a wavelength reference. We began with an iodine-free spectrum of HIP 428, oversampled onto a grid with resolution $15 \mathrm{~m} \mathrm{~s}^{-1}$. For each observation, we restricted our analysis to the 16 orders covered by the "green" CCD chip, which covers the region typically used in iodine cell analyses, as well as the first two orders covered by the "red" chip where telluric contamination is negligible. From these 18 orders, we first estimated and divided out the continuum flux level following the method of Pineda et al. (2013). We then removed the regions of the spectrum contaminated by telluric lines. We added to this template a shifted, scaled version of itself to represent LHS $6343 \mathrm{~B}$. We varied the positions of both stars and compared to the observed spectrum of LHS 6343 in order to find the maximum likelihood velocity separation between the two stars. By assuming the relative RV of LHS 6343 B does not change over our observing baseline, our method enables us to measure the RV of LHS 6343 A relative to that of a stationary wavelength calibration source observed simultaneously.

There is no evidence of orbital motion of LHS 6343 B at the level of our RV precision. From an observed projected separation and mass estimate we can estimate the maximum expected RV acceleration induced by a companion. Following Torres (1999) and Knutson et al. (2014), the maximum RV acceleration is
Table 1

Radial Velocities for LHS 6343

\begin{tabular}{|c|c|c|}
\hline JD -2440000 & $\begin{array}{c}\mathrm{RV} \\
\left(\mathrm{km} \mathrm{s}^{-1}\right)\end{array}$ & $\begin{array}{c}\text { Uncertainty } \\
\left(\mathrm{km} \mathrm{s}^{-1}\right)\end{array}$ \\
\hline 15373.095 & 12.993 & 0.498 \\
\hline 15373.998 & 13.878 & 0.429 \\
\hline 15377.078 & 3.041 & 0.425 \\
\hline 15377.098 & 2.825 & 0.423 \\
\hline 15378.030 & -2.470 & 0.562 \\
\hline 15379.052 & -4.599 & 0.076 \\
\hline 15380.127 & -5.967 & 0.082 \\
\hline 15380.827 & -5.412 & 0.089 \\
\hline 15380.831 & -5.015 & 0.166 \\
\hline 15395.984 & 3.726 & 0.084 \\
\hline 15396.970 & 8.522 & 0.068 \\
\hline 15404.974 & -5.447 & 0.092 \\
\hline 15405.821 & -5.618 & 0.074 \\
\hline 15406.865 & -3.860 & 0.086 \\
\hline 15407.853 & -0.495 & 0.666 \\
\hline 15413.032 & 11.540 & 0.072 \\
\hline 15414.009 & 7.951 & 0.089 \\
\hline 15668.120 & 8.714 & 0.161 \\
\hline 15669.083 & 4.243 & 0.174 \\
\hline 15673.982 & -3.661 & 0.083 \\
\hline 15705.917 & 10.005 & 0.093 \\
\hline 15843.859 & 13.444 & 0.084 \\
\hline 16116.017 & -3.562 & 0.077 \\
\hline 16164.014 & 8.408 & 0.064 \\
\hline 16172.915 & 10.070 & 0.078 \\
\hline 16192.886 & -4.885 & 0.073 \\
\hline 16498.042 & -5.035 & 0.079 \\
\hline 16506.891 & 9.963 & 0.073 \\
\hline 16513.001 & -3.995 & 0.081 \\
\hline 16513.988 & 0.033 & 0.733 \\
\hline 16522.939 & -3.889 & 0.078 \\
\hline 16524.890 & -5.555 & 0.113 \\
\hline 16524.892 & -5.473 & 0.081 \\
\hline 16530.943 & 13.348 & 0.092 \\
\hline
\end{tabular}

defined such that

$$
|\dot{v}|<68.8 \mathrm{~m} \mathrm{~s}^{-1} \mathrm{yr}^{-1}\left(\frac{M_{\text {comp }}}{M_{\text {Jup }}}\right)\left(\frac{d}{\mathrm{pc}} \frac{\rho}{\operatorname{arcsec}}\right)^{-2},
$$

for a system at a distance $d$, with a companion with mass $M_{\text {comp }}$ at an angular separation $\rho$. For a companion with a mass approximately $30 \%$ of the Sun's and a projected separation $(d \rho)$ of approximately $20 \mathrm{AU}$, we expect a maximum RV acceleration of $40 \mathrm{~m} \mathrm{~s}^{-1} \mathrm{yr}^{-1}$. We would only observe this RV acceleration if we happened to observe the two stars at the time of their maximum orbital separation and if their orbit was edge-on to our line of sight. Our RV signal is considerably larger than any effects induced by LHS 6343 B; any RV acceleration over our $3 \mathrm{yr}$ baseline is similar in size to our measurement uncertainties.

The median RV precision of our observations is $85 \mathrm{~m} \mathrm{~s}^{-1}$. Our RV precision is much lower $\left(\approx 400 \mathrm{~m} \mathrm{~s}^{-1}\right)$ for the first four observations when the spectra are contaminated by the iodine cell. Our RV precision is also impeded when the difference between the RV of LHS 6343 A and LHS 6343 B is smaller than one-half of a pixel, about $500 \mathrm{~m} \mathrm{~s}^{-1}$.

A table of our RVs is included as Table 1.

\subsection{Visible-light Adaptive Optics Imaging}

J11 estimated the third light contribution of LHS 6343 B in the Kepler bandpass by extrapolating from $J H K$ adaptive 


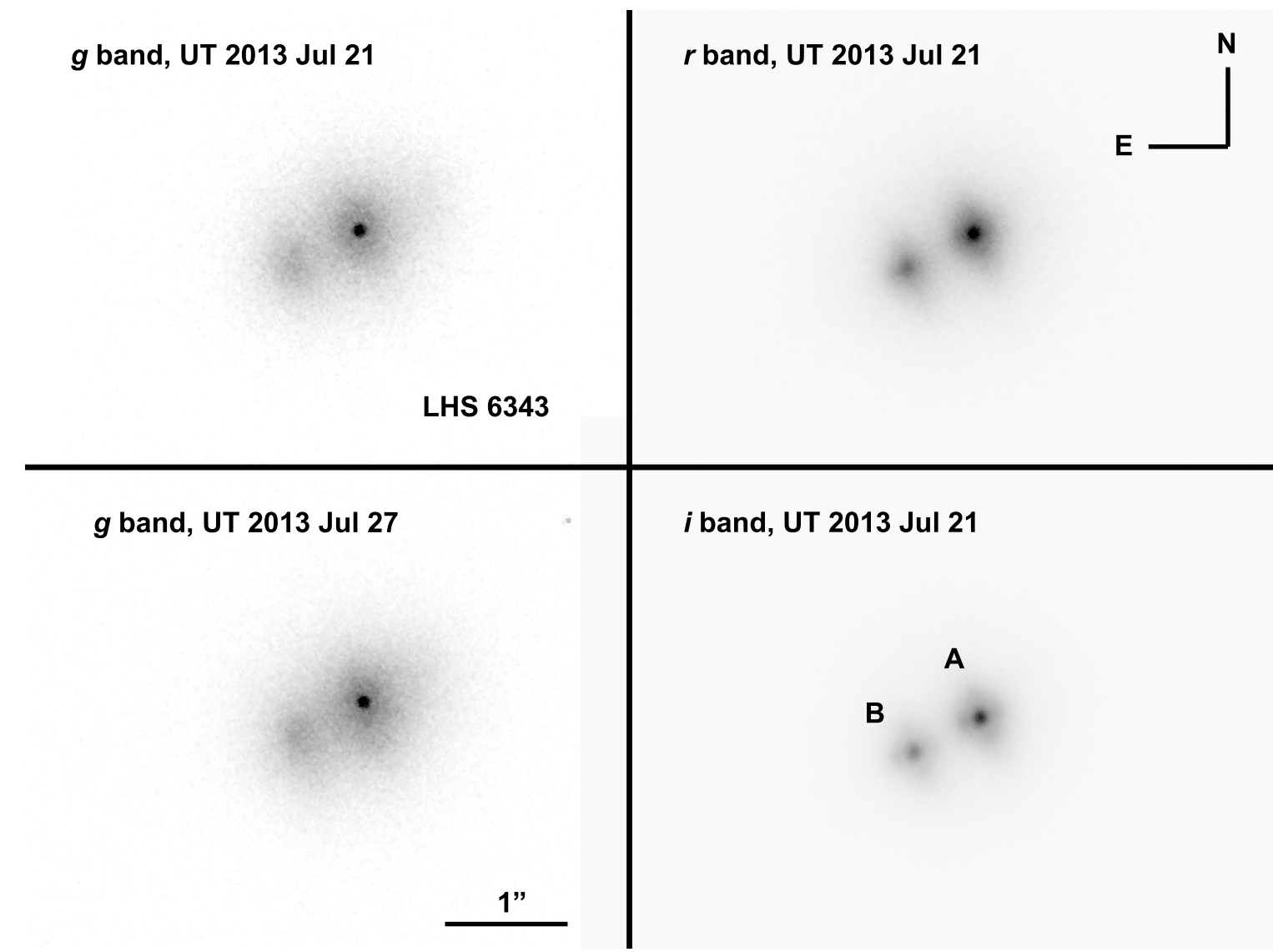

Figure 1. Robo-AO adaptive optics imaging of the LHS 6343 system taken with three different bandpasses. Both the scale and orientation are held constant across all images. We obtained two images of the system in the $g$ band, six days apart. We obtained a single image of the system in both the $r$ and $i$ bands.

optics observations using the Padova model atmospheres of Girardi et al. (2002). To minimize any potential biases that may be induced by their reliance on stellar models, we obtained adaptive optics imaging of LHS 6343 with the Robo-AO laser adaptive optics and imaging system on the Palomar Observatory 60 inch telescope (Baranec et al. 2014). Robo-AO successfully observed thousands of KOIs; we used their standard setup (Law et al. 2014). With Sloan Digital Sky Survey $g, r$, and $i$ filters (York et al. 2000), we imaged the system on UT 2013 July 21; we observed the system again in $g$ band on UT 2013 July 27. Each observation consisted of full-frame-detector readouts at $8.6 \mathrm{~Hz}$ for $90 \mathrm{~s}$. We use $100 \%$ of the frames during each integration. The images were then combined using a shift-andadd processing scheme, using LHS $6343 \mathrm{~A}$ as the tip-tilt star. At all wavelengths, we detected both LHS 6343 A and LHS 6343 B, as shown in Figure 1. While we would be sensitive to a change in the position angle between the two $\mathrm{M}$ dwarfs of two degrees, we do not detect any orbital motion of LHS $6343 \mathrm{~B}$ relative to LHS 6343 A between the original Palomar/PHARO data in 2010 and these observations in 2013.

To calculate the relative flux ratio of the two stars in each bandpass, we sky-subtract our observations and measure the flux inside a 0.5 aperture centered on each star. The pointspread functions of each star are larger than the apertures, so each aperture contains light from both stars. We subtract out the contamination from each star by measuring the flux in a similar aperture on the opposite side of each star.

In our $g$-band data we observed tripling, induced when the shift-and-add processing algorithm temporarily locks on LHS $6343 \mathrm{~B}$ instead of LHS 6343 A. Tripling causes the appearance of an artificial third object coaxial with the two real objects. The third object is observed to have the same projected separation between the primary as the true secondary, at a position angle offset of $180^{\circ}$, as discussed by Law et al. (2006). By measuring the flux ratios between the primary star and the two imaged companions, and defining $I_{j k} \equiv F_{j} / F_{k}$, then the true binary flux ratio $F_{R}$ is

$$
F_{R}=\frac{2 I_{13}}{I_{12} I_{13}+\sqrt{I_{12}^{2} I_{13}^{2}-4 I_{12} I_{13}}},
$$

where $F_{1}$ is the observed flux from the primary component, $F_{2}$ the observed flux from the secondary component, and $F_{3}$ the observed light from the tertiary, "tripled" component. When $F_{3}=0$ this equation is undefined, but the asymptotic behavior is correct.

We find the third light contributions in each bandpass are given such that $\Delta g=0.93 \pm 0.07, \Delta r=0.74 \pm 0.06$, and $\Delta i=$ $0.57 \pm 0.05$. From these, we interpolate using the Dartmouth stellar models to calculate a value for the third light in the Kepler bandpass, which encompasses roughly the $g, r$, and $i$ filters. We find $\Delta K_{p}=0.71 \pm 0.07 \mathrm{mag}$. This is consistent with the extrapolation of J11, who predict a third light in the Kepler bandpass of $\Delta K_{p}=0.74 \pm 0.10$.

\subsection{NIR Spectroscopy}

The transit light curve itself can be used to measure some properties of LHS $6343 \mathrm{~A}$, such as the stellar density. Other parameters such as the stellar temperature, as well as all 


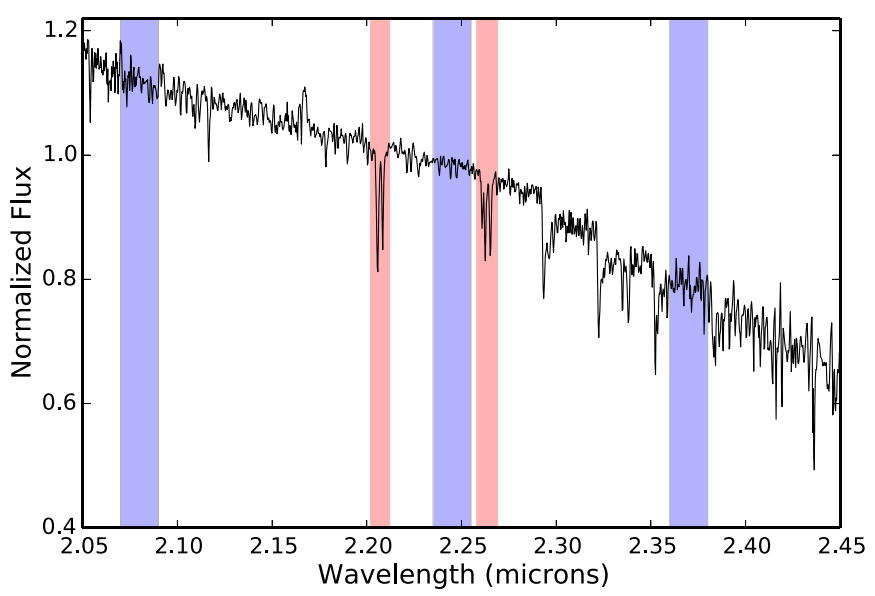

Figure 2. Combined-light $K$-band spectrum for the LHS 6343 system. The broad, blue shaded regions are used to derive the " $\mathrm{H}_{2} \mathrm{O}-\mathrm{K} 2$ water index," as described in Section 3.1. The narrow, red shaded regions encompass the sodium doublet and calcium triplet. Together, these regions have been used to develop empirical relations for the temperature and metallicity of M dwarfs (Rojas-Ayala et al. 2012).

physical properties of LHS $6343 \mathrm{~B}$, can only be estimated by relying on stellar models. To inform the models, on UT 2012 July 5 we obtained simultaneous $J H K$ spectroscopy with the TripleSpec Spectrograph on the 200" Hale Telescope at Palomar Observatory. TripleSpec is a near-IR slit spectrograph with a resolving power $(\lambda / \Delta \lambda)$ of 2700 (Wilson et al. 2004; Herter et al. 2008).

Observations were collected on four positions along the slit, $\mathrm{ABCD}$, to minimize the effects of hot and dead pixels on the spectrograph detector. Each exposure was $30 \mathrm{~s}$ long in order to achieve a signal-to-noise ratio of 60 . We then observed a nearby, rapidly rotating $\mathrm{A} 0 \mathrm{~V}$ star to calibrate absorption lines caused by the Earth's atmosphere.

To reduce the data, we followed the methodology of Muirhead et al. (2014), using the SpexTool reduction package of Cushing et al. (2004). We differenced the A and B observations and the $\mathrm{C}$ and D observations separately, then extracted the combinedlight spectrum and combined the separate observations with SpexTool. To remove the system's absolute RV of $-46 \mathrm{~km} \mathrm{~s}^{-1}$, we cross-correlated the spectrum with data from the IRTF spectral library (Cushing et al. 2005; Rayner et al. 2009), then applied an offset to the wavelength solution corresponding to the peak of the cross-correlation function. The result is a single spectrum displaying the combined light from LHS 6343 A and LHS 6343 B, as shown in Figure 2.

\section{DATA ANALYSIS}

\subsection{Temperature and Metallicity of LHS $6343 A$ and B}

We measured the temperature of each star following the method of Rojas-Ayala et al. (2012), who built on the efforts of Covey et al. (2010) to determine a relation between $K$-band spectroscopic features and the temperature and metallicity of M dwarfs. Specifically, Rojas-Ayala et al. define a temperaturesensitive " $\mathrm{H}_{2} \mathrm{O}-\mathrm{K} 2$ water index," representing the water opacity between $2.07 \mu \mathrm{m}$ and $2.38 \mu \mathrm{m}$ :

$$
\mathrm{H}_{2} \mathrm{O}-\mathrm{K} 2=\frac{\langle\mathcal{F}(2.070-2.090)\rangle /\langle\mathcal{F}(2.235-2.255)\rangle}{\langle\mathcal{F}(2.235-2.255)\rangle /\langle\mathcal{F}(2.360-2.380)\rangle} .
$$

Here, $\langle\mathcal{F}(a-b)\rangle$ represents the median flux level in the region $[a, b]$, with both $a$ and $b$ in $\mu \mathrm{m}$. They also defined

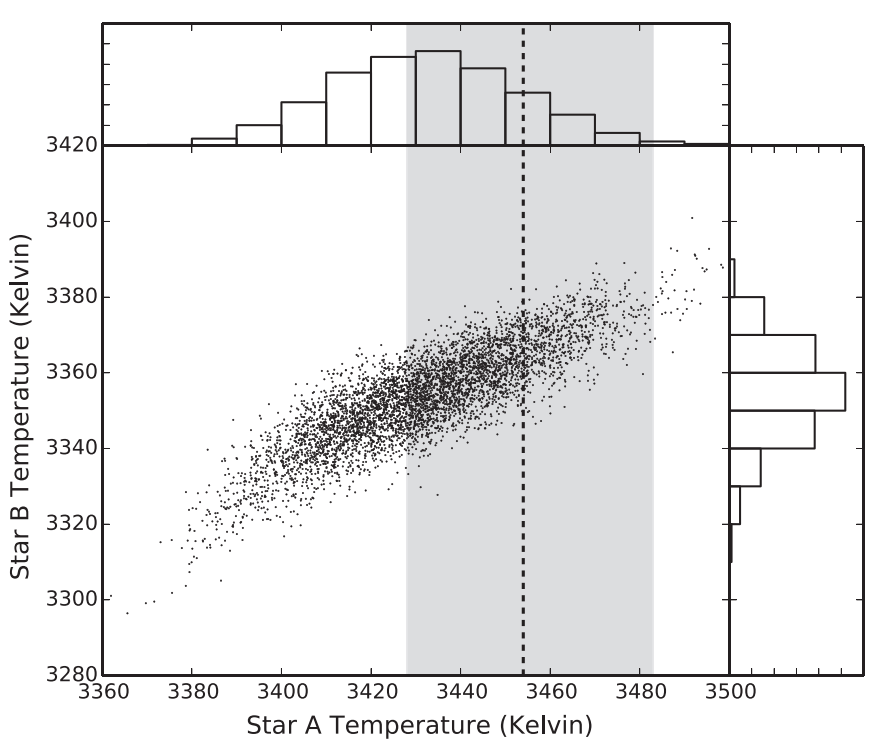

Figure 3. Joint posterior on the effective temperature of LHS $6343 \mathrm{~A}$ and LHS $6343 \mathrm{~B}$. Marginalizing over the temperature of each star separately, we find the A component has a temperature of $3431 \pm 21 \mathrm{~K}$ and the B component has a temperature of $3354 \pm 17 \mathrm{~K}$. The dashed line and shaded region correspond to the temperature of LHS 6343 A expected based on our model-independent mass measurement from the combined transit and RV fit.

a relation between a star's metallicity, the $\mathrm{H}_{2} \mathrm{O}-\mathrm{K} 2$ index, and the equivalent width of the $2.21 \mu \mathrm{m}$ sodium doublet and $2.26 \mu \mathrm{m}$ calcium triplet. We calculated $\mathrm{H}_{2} \mathrm{O}-\mathrm{K} 2$ and the two equivalent widths, as well as their uncertainties, by creating a sequence of simulated spectra in which random noise is added to the observed flux consistent with the flux uncertainty at each wavelength. We found the calculated $\mathrm{H}_{2} \mathrm{O}-\mathrm{K} 2$ values to be normally distributed such that $\mathrm{H}_{2} \mathrm{O}-\mathrm{K} 2=0.919 \pm 0.002$. The equivalent width of the sodium doublet is $5.533 \pm 0.101 \AA$ and the equivalent width of the calcium triplet is $3.863 \pm 0.089 \AA$.

If our spectrum consisted of the flux from only one star, we could convert our value directly into a stellar effective temperature and metallicity. In this case, each value is really the combination of two separate values, one for each $\mathrm{M}$ dwarf. However, if we assume the two stars have the same metallicity, useful information can still be extricated. We first drew from the posterior of $\Delta K$ values from our PHARO near-IR adaptive optics observations and our posteriors for $\mathrm{H}_{2} \mathrm{O}-\mathrm{K} 2$ and the equivalent widths. From these, we used the relations of Rojas-Ayala et al. (2012) to calculate the system metallicity. We then interpolated the table provided in that paper to find a relation between $\mathrm{H}_{2} \mathrm{O}-\mathrm{K} 2$ and effective temperature for a given metallicity. Using the Dartmouth stellar evolution models, we then determined which two modeled stars best fit both the observed flux ratio and combined $\mathrm{H}_{2} \mathrm{O}-\mathrm{K} 2$ index value. By repeating this process many times, continuously drawing from the posteriors for each measured value we determined a posterior on the temperature, and by extension the mass, of each star. The joint posterior on the temperature of the two stars is shown as Figure 3.

\subsection{Transit Parameters}

To measure the parameters of LHS $6343 \mathrm{C}$, we forward modeled the LHS 6343 A-C system over the timespan from the launch of Kepler to the date of the final RV observation in 2013. At each time corresponding to an RV observation, we calculated the expected RV relative to a stationary LHS 6343 B assuming a Keplerian orbit. At each Kepler timestamp during a transit or 


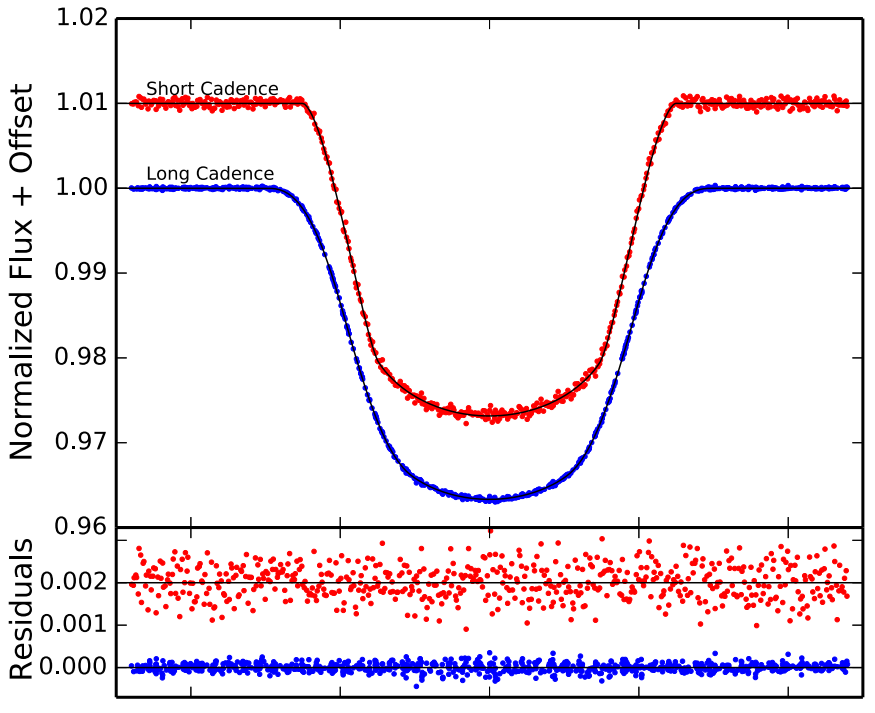

Time from Transit Center (hours)

Figure 4. Phase-folded transit light curve, fit to the maximum likelihood model. Blue points represent long cadence data and red points represent short cadence data. The scale of the residuals is a factor of five larger than the scale of the light curve.

near the expected time of secondary eclipse, we calculated the expected relative flux assuming a Mandel \& Agol (2002) light curve model. We fit four limb darkening parameters using the prescription of Claret \& Bloemen (2011), allowing the value for each limb darkening coefficient to float as a free parameter. In calculating the light curves, we used an adapted version of the PyAstronomy package, ${ }^{9}$ modified to allow eccentric orbits.

In all, we fit for 16 parameters: $\sqrt{e} \cos \omega, \sqrt{e} \sin \omega$, time of central transit, orbital period, brown dwarf mass, orbital inclination, LHS $6343 \mathrm{~A}-\mathrm{C}$ radius ratio, four limb darkening parameters, the third light from LHS $6343 \mathrm{~B}, \log (g)$ of LHS $6343 \mathrm{~A}$, the secondary eclipse depth, the stellar mass, and the RV zero point (relative to LHS 6343 B). We did not use an RV jitter term, as our $\mathrm{RV}$ uncertainties of $\sim 100 \mathrm{~m} \mathrm{~s}^{-1}$ are significantly larger than the jitter expected for a main-sequence $\mathrm{M}$ dwarf. We used emcee, an affine-invariant ensemble sampler described by Goodman $\&$ Weare (2010) and implemented by Foreman-Mackey et al. (2013), to maximize the likelihood function:

$$
\begin{aligned}
\mathcal{L}= & 0.5\left[\sum_{i}\left(\frac{\mathrm{RV}_{\text {model }, i}-\mathrm{RV}_{\text {observed }, i}}{\sigma_{\mathrm{RV}, i}}\right)^{2}\right. \\
& +\sum_{i}\left(\frac{f_{\text {model SC }, i}-f_{\text {observed SC }, i}}{\sigma_{f_{\mathrm{SC}}, i}}\right)^{2} \\
& \left.+\sum_{i}\left(\frac{f_{\text {model LC }, i}-f_{\text {observed LC }, i}}{\sigma_{f_{\mathrm{LC}}, i}}\right)^{2}\right] .
\end{aligned}
$$

Here, $f_{\mathrm{LC}}$ corresponds to the observed flux in the Kepler long cadence data and $f_{\mathrm{SC}}$ corresponds to the short cadence data. The period we fit and report here is the period observed in the frame of an observer at the barycenter of the solar system, not in the frame of the LHS 6343 system. That is, we do not correct for relativistic effects induced by the star system's systemic velocity.

We imposed two different priors on the stellar mass, reflecting various levels of trust in theoretical stellar evolutionary models.

\footnotetext{
9 https://github.com/sczesla/PyAstronomy
}

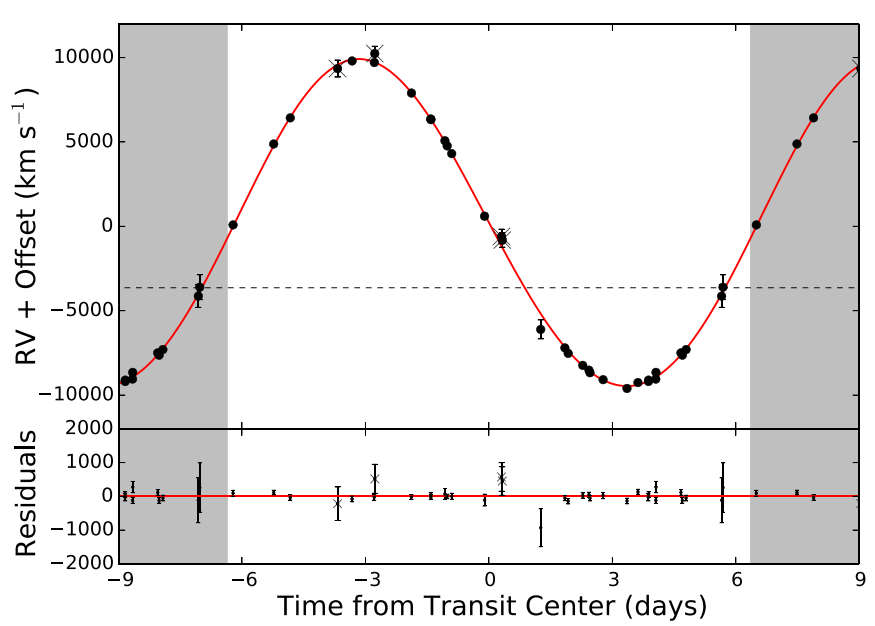

Figure 5. Phase-folded RV data curve, fit to the maximum likelihood model. For the majority of observations, the data points are larger than the size of the error bars. The gray shaded regions represent an extension of the RV data beyond one phase to provide clarity for the reader. Observations marked with an cross represent data collected while using the iodine cell. The dashed line represents the RV of LHS 6343 B, which does not change at the level of our precision over the 3 yr RV baseline.

First we apply the stellar empirical mass-radius relation of Boyajian et al. (2012), which encodes no direct model-dependent information, as a prior We use their relation for "single stars." While our star has a wide binary companion at tens of AU, the single collection is more representative of LHS 6343 A than the short-period eclipsing binaries used to build the eclipsing binary main sequence of Boyajian et al. (2012). Given a precise measurement of the stellar density $\rho_{\star}$, semimajor axis $a / R_{\star}$, Doppler semiamplitude $K$, eccentricity, and inclination, the mass and radius of both the primary and secondary star can then be calculated. We derive these relations in the Appendix.

We next repeated this analysis, applying a prior on the stellar mass using the spectroscopic parameters from our TripleSpec analysis, as described in Section 3.1.

In each of these cases, we can calculate the mass and radius of LHS $6343 \mathrm{~B}$ through the Dartmouth models by comparing the relative brightness of LHS 6343 A and LHS 6343 B in conjunction with the (now known) mass of LHS $6343 \mathrm{~A}$. We can also measure a model-dependent distance to the system, which depends both on our measured mass and the mass-luminosity relation encoded in the stellar models.

The best-fit model to the light curve data and RVs are plotted in Figures 4 and 5, respectively.

\section{RESULTS}

The orbital parameters for LHS 6343 C are listed in Table 2. The physical properties of the LHS 6343 system are listed in Table 3. In the latter table, we include two columns of values. The first set of values represents the values we find using our data-driven model, using only the empirical mass-radius relation of Boyajian et al. (2012) without any direct use of stellar models. The second set of values corresponds to the inclusion of a model-dependent prior on the stellar mass. In this case, we impose as a prior our mass derived from the near-IR spectroscopy, found in Section 3.1.

We find that we are able to measure the observed transit depth, uncorrected for the third light contributions of LHS $6343 \mathrm{~B}$, to a precision of $0.5 \%$. We are additionally able to measure the Doppler semiamplitude $K$ to $0.3 \%$. Therefore, our uncertainties 
Table 2

Orbital Parameters for the LHS 6343 AC System

\begin{tabular}{|c|c|c|c|}
\hline Parameter & Value & & $\begin{array}{c}1 \sigma \text { Confidence } \\
\text { Interval }\end{array}$ \\
\hline Orbital period, $P$ (days) & 12.7137941 & \pm & 0.0000002 \\
\hline Transit center (TDB -2440000) & 15008.07259 & \pm & 0.00001 \\
\hline Radius ratio, $\left(R_{P} / R_{\star}\right)$ & 0.216 & \pm & 0.004 \\
\hline Observed transit depth (\%) & 3.198 & \pm & 0.015 \\
\hline Scaled semimajor axis, $a / R_{\star}$ & 46.0 & \pm & 0.4 \\
\hline Orbital inclination, $i$ (deg) & 90.45 & \pm & 0.03 \\
\hline Transit impact parameter, $b$ & 0.36 & \pm & 0.02 \\
\hline Argument of periastron $\omega(\mathrm{deg})$ & -40 & \pm & 4 \\
\hline Eccentricity & 0.030 & \pm & 0.002 \\
\hline Secondary phase $(e \cos \omega)$ & 0.0228 & \pm & 0.0008 \\
\hline Secondary depth (ppm) & 25 & \pm & 7 \\
\hline Velocity semiamplitude $K_{A}\left(\mathrm{~km} \mathrm{~s}^{-1}\right)$ & 9.69 & \pm & 0.02 \\
\hline Star A-B RV offset $\left(\mathrm{km} \mathrm{s}^{-1}\right)$ & 3.64 & \pm & 0.02 \\
\hline
\end{tabular}

Note. All parameters calculated by simultaneously fitting to the RV data and Kepler data near the times of transit and secondary eclipse.

in the brown dwarf's physical parameters are dominated by the uncertainties on the absolute physical parameters of the two $\mathrm{M}$ dwarfs in the system.

We can measure the stellar mass directly from the light curve and RV observations without any direct reliance on theoretical stellar models, as shown in the Appendix. In this case, we measure a mass for LHS $6343 \mathrm{~A}$ of $0.381 \pm 0.019 M_{\odot}$ and a radius of $0.380 \pm 0.007 R_{\odot}$. We then find a mass and radius of LHS $6343 \mathrm{C}$ of $64.6 \pm 2.1 M_{\text {Jup }}$ and $0.798 \pm 0.014 R_{\text {Jup }}$, respectively. Thus, in this case we can measure the mass of the brown dwarf to a precision of $3.2 \%$ and the radius to $1.8 \%$.

From our near-IR spectroscopic analysis of the system, we measure a temperature for LHS 6343 A of $3431 \pm 21 \mathrm{~K}$, which gives us a mass of $0.339 \pm 0.016 M_{\odot}$. We repeat our analysis, using this value as a prior on our stellar mass. In this case, we find a value for the stellar mass between our empirical value and that imposed by our model prior: $0.358 \pm 0.011 M_{\odot}$. We then find a mass for the brown dwarf of $62.1 \pm 1.2 M_{\mathrm{Jup}}$ and a radius of $0.782 \pm 0.013 R_{\mathrm{Jup}}$. This is a model-dependent mass measured to a precision of $1.9 \%$ and a model-dependent radius to $1.4 \%$.

Our brown dwarf mass is consistent with that found by $\mathrm{J} 11$, while our radius is smaller at the $1.4 \sigma$ level. Part of this discrepancy may be due to the choice of models used: these authors used the Padova model grids of Girardi et al. (2002). These models predict a larger mass than both the Dartmouth models we use and the BT-Settl models (Allard \& Freytag 2010). Using the Padova models, the authors of the discovery paper adopted a slightly smaller $\log (g)$, which for a given mass implies a larger star, and therefore a larger planet. The discrepancy may also be affected by our choices of limb darkening models: the authors of the discovery paper use a quadratic limb darkening model. With only five transits observed, this is a reasonable choice. Given the signal to noise obtained from fitting four years of Kepler data simultaneously, we require a four-parameter limb darkening solution to develop an appropriate model fit.

Our mean density for LHS 6343 C is $40 \%$ larger than that reported in the discovery paper. This appears to be because the authors of that paper misreported their density, as it is inconsistent with their reported mass and radius. These authors may have reported the density relative to Jupiter, not in units of $\mathrm{g} \mathrm{cc}^{-1}$ as listed in their Table 5. Even with this correction, the density we report is larger than the density of J11 due to the difference in the radius of the brown dwarf described in the previous paragraph.

We measure a period of $12.7137941 \pm 0.0000002$ days in the frame of the solar system. The uncertainty in the period is $17 \mathrm{~ms}$, and the period is measured to a precision of 15 parts per billion.

Table 3

Physical Parameters for LHS 6343 ABC

\begin{tabular}{|c|c|c|c|c|c|c|c|}
\hline Parameter & $\begin{array}{c}\text { Value } \\
\text { (Empirical Prior) }\end{array}$ & & $\begin{array}{c}1 \sigma \text { Confidence } \\
\text { Interval }\end{array}$ & $\begin{array}{c}\text { Value } \\
\text { (Model Prior) }\end{array}$ & & $\begin{array}{c}1 \sigma \text { Confidence } \\
\text { Interval }\end{array}$ & Comment \\
\hline \multicolumn{8}{|c|}{ Stellar parameters } \\
\hline$M_{A}\left(M_{\odot}\right)$ & 0.381 & \pm & 0.019 & 0.358 & \pm & 0.011 & A \\
\hline$M_{B}\left(M_{\odot}\right)$ & & & & 0.292 & \pm & 0.013 & A \\
\hline$R_{A}\left(R_{\odot}\right)$ & 0.380 & \pm & 0.007 & 0.373 & \pm & 0.005 & A \\
\hline$R_{B}\left(R_{\odot}\right)$ & & & & 0.394 & \pm & 0.012 & A \\
\hline$\rho_{A}\left(\rho_{\odot}\right)$ & 6.96 & \pm & 0.19 & 6.93 & \pm & 0.19 & A \\
\hline $\log g_{A}(\mathrm{cgs})$ & 4.86 & \pm & 0.01 & 4.85 & \pm & 0.01 & A \\
\hline Metallicity $[\mathrm{Fe} / \mathrm{H}]$ & & & & 0.03 & \pm & 0.26 & B \\
\hline Metal content $[\mathrm{a} / \mathrm{H}]$ & & & & 0.02 & \pm & 0.19 & B \\
\hline Distance $(\mathrm{pc})$ & & & & 32.7 & \pm & 1.3 & $\mathrm{C}$ \\
\hline Flux ratio $F_{B} / F_{A}, K_{p}$ & 0.461 & \pm & 0.055 & 0.518 & \pm & 0.032 & A \\
\hline$\Delta K_{p}(\mathrm{mag})$ & 0.84 & \pm & 0.12 & 0.71 & \pm & 0.07 & A \\
\hline$T_{\mathrm{eff}, A}(\mathrm{~K})$ & & & & 3431 & \pm & 21 & $\mathrm{~B}$ \\
\hline$T_{\mathrm{eff}, B}(\mathrm{~K})$ & & & & 3354 & \pm & 17 & B \\
\hline \multicolumn{8}{|c|}{ Brown dwarf parameters } \\
\hline$M_{C}\left(M_{\mathrm{Jup}}\right)$ & 64.6 & \pm & 2.1 & 62.1 & \pm & 1.2 & A \\
\hline$R_{C}\left(R_{\mathrm{Jup}}\right)$ & 0.798 & \pm & 0.014 & 0.783 & \pm & 0.011 & A \\
\hline Semimajor axis, A-C system (AU) & 0.0812 & \pm & 0.0013 & 0.0797 & \pm & 0.0008 & A \\
\hline Mean planet density, $\rho_{C}\left(\mathrm{~g} \mathrm{~cm}^{-3}\right)$ & 170 & \pm & 5. & 173 & \pm & 5 & A \\
\hline $\log g_{C}(\mathrm{cgs})$ & 5.419 & \pm & 0.008 & 5.420 & \pm & 0.008 & A \\
\hline$T_{\mathrm{eq}}\left(T_{\mathrm{eff}}\left(R_{\star} / 2 a\right)^{1 / 2}\right)(\mathrm{K})$ & & & & 358 & \pm & 3 & $\mathrm{~A}, \mathrm{~B}$ \\
\hline
\end{tabular}

Notes. (A) Calculated by simultaneously fitting to the RV data and Kepler data near the times of transit and secondary eclipse. (B) Measured from near-IR spectroscopy following the method of Rojas-Ayala et al. (2012). (C) Calculated by fitting the observed apparent magnitudes to model-predicted absolute magnitudes. 


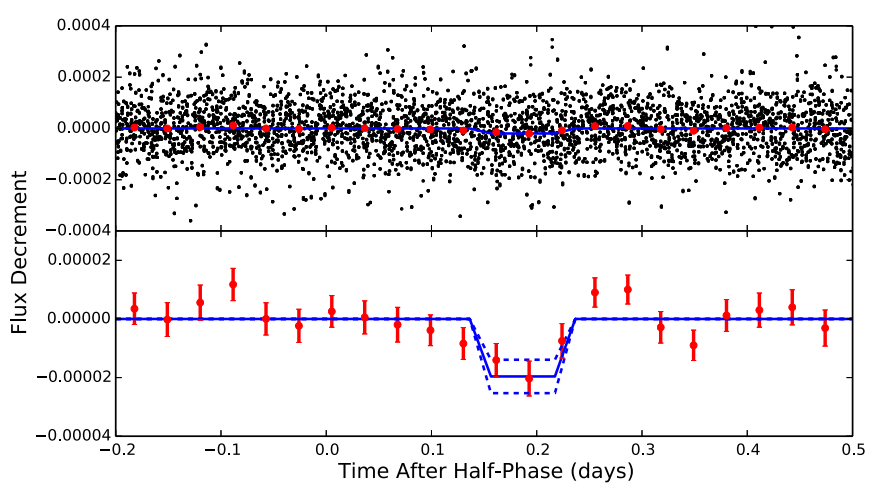

Figure 6. Secondary eclipse of LHS $6343 \mathrm{C}$ as observed by Kepler. Top: in black, the Kepler data are phase-folded and plotted; we bin every 0.03 days of observations together to reduce the apparent scatter, as shown in red. As the noise is nearly completely white, this is justified for plotting purposes. In blue is our best-fitting secondary eclipse model. We treat the brown dwarf as a uniform sphere in our modeling efforts. Bottom: same as the above, excluding the raw data. We detect an eclipse depth of $25 \pm 7 \mathrm{ppm}$ after accounting for the correction for the third light contribution from LHS $6343 \mathrm{~B}$. The dashed blue lines represent the $1 \sigma$ deviation in eclipse depth from the best-fitting model.

We measure the total mass in the LHS 6343 AC system to a precision of $4.8 \%$. Neglecting our uncertainty in the measured period, from differentiating Kepler's Third Law we expect our measurement of the semimajor axis to be three times more precise than that of the total mass. In fact, we measure a semimajor axis of $0.0812 \pm 0.0013 \mathrm{AU}$, a precision of $1.6 \%$.

\subsection{Secondary Eclipse Observation}

J11 do not detect a secondary eclipse and can only place an upper limit of $65 \mathrm{ppm}$ on the potential eclipse depth. With a full $4 \mathrm{yr}$ of Kepler data, we are considerably more sensitive to eclipses. From the RVs and shape of the primary eclipse alone, we know the A-C system has a nonzero eccentricity: we find $e \cos \omega=0.024 \pm 0.003$. As a result, we expect the secondary eclipse to occur approximately $4.5 \mathrm{hr}$ after the midpoint between consecutive primary transits.

When we include a secondary eclipse in our system model, we detect a signal at $3.5 \sigma$, as shown in Figure 6 . This eclipse has a depth of $25 \pm 7 \mathrm{ppm}$ and occurs $4.44 \pm 0.16 \mathrm{hr}$ after the midpoint between primary transits. From these data, we measure $e \cos \omega=0.0228 \pm 0.0008$.

\subsection{Distance to the LHS 6343 System}

There is, at present, no measured parallax to the LHS $6343 \mathrm{C}$ system. We must therefore rely on stellar models to convert the measured apparent magnitudes to distance estimates. J11, using the Padova model atmospheres, announced a distance to the system of $36.6 \pm 1.1 \mathrm{pc}$. The Dartmouth models predict a lower mass, and therefore a lower luminosity for LHS $6343 \mathrm{~A}$, so to maintain the observed brightness of the system from $g$ to $K_{s}$ band, these models require a smaller distance modulus. We find a model-dependent distance to the system of $32.7 \pm 1.3 \mathrm{pc}$. A measured parallax to this system, either from the ground or from Gaia, will be useful for resolving the $2 \sigma$ discrepancy between these distances, informing the upcoming next generation of stellar evolution models.

\section{DISCUSSION}

There are now nine brown dwarfs with measured masses and radii (Moutou et al. 2013). Of this sample, there are only four

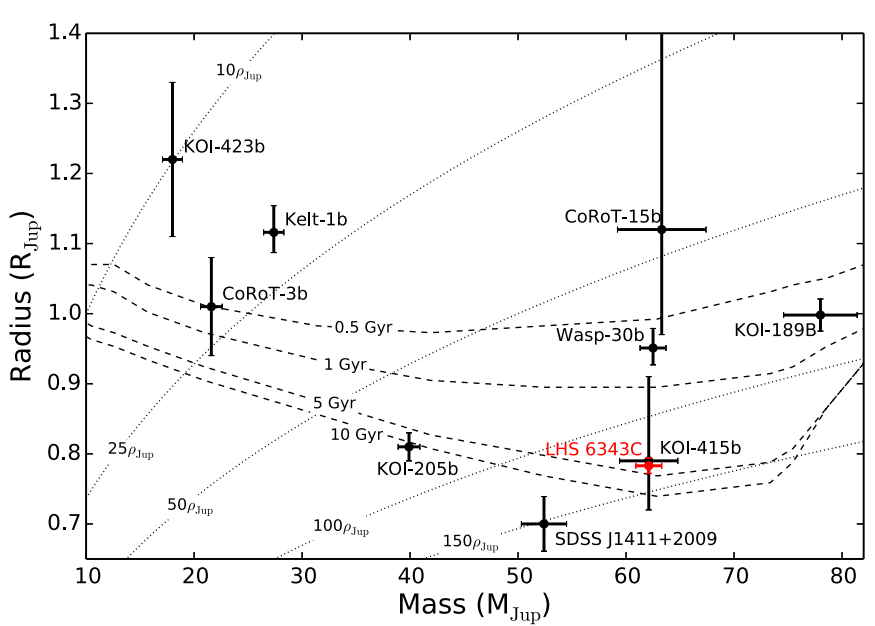

Figure 7. Mass-radius diagram for known transiting brown dwarfs. The dashed lines represent the Baraffe et al. (2003) isochrones for (top to bottom) ages of 0.5 , 1.0, 5.0, and 1.0 billion years. The dotted lines are isodensity contours for (top left to bottom right) densities corresponding to $10,25,50,100$, and 150 times the density of Jupiter. LHS $6343 \mathrm{C}$ has a density of $130 \pm 4 \rho_{\text {Jup }}$ and appears to have an age of 3-5 Gyr. Data taken from Deleuil et al. (2008), Bouchy et al. (2011b, 2011a), Siverd et al. (2012), Díaz et al. (2013, 2014), Moutou et al. (2013), Triaud et al. (2013), and Littlefair et al. (2014). Not shown are the components of the young binary brown dwarf system 2MASS 2053-05 (Stassun et al. 2006), which have radii well above the plot range.

that are not inflated due to youth or irradiation. LHS $6343 \mathrm{C}$ is effectively a field brown dwarf: the equilibrium temperature for an object at its orbital separation is $360 \mathrm{~K}$ while a $65 M_{\text {Jup }}$ brown dwarf is expected to cool to only $700 \mathrm{~K}$ over a Hubble time (Burrows et al. 2001). Thus, the irradiation from the primary star on the brown dwarf is negligible. Additionally, since the system has a nonzero eccentricity, the system is not tidally locked, minimizing any effects the primary star may have on any one point on the brown dwarf's surface. LHS $6343 \mathrm{C}$ can be used as a laboratory to study the physics of solitary brown dwarfs, as it is effectively a field brown dwarf with a known mass, radius, and metallicity. The sample of transiting brown dwarfs that can be used to probe the physics of field brown dwarfs is highly limited, making each individual system extremely valuable.

There is some evidence that our current best understanding of the physics of brown dwarfs is incomplete. Dupuy et al. (2009) find evidence for a "substellar luminosity problem," in which the brown dwarf binary HD 130948 BC is twice as luminous as predicted by evolutionary models. A similar result is found in the G1 417 BC system (Dupuy et al. 2014). As these are the only two brown dwarf systems with reliable measurements of both mass and age, this result is suggestive of a fundamental issue with substellar models.

We have only a lower limit on the age of the system: J11 find no youth indicators present in the LHS 6343 system so it is likely not less than 1-2 Gyr old. Therefore, a measured luminosity would be most useful as a probe of this specific plane if the luminosity were consistent with extreme youth $(<1 \mathrm{Gyr})$ or extreme age ( $>14 \mathrm{Gyr})$. A measured luminosity is still useful, as it allows us to locate the brown dwarf's position in the mass-radius-luminosity plane. While there is a collection of non-inflated brown dwarfs with masses and luminosities measured, there are only three with mass and radius and none with both radius and luminosity. Moreover, we also know the metallicity of the brown dwarf, assuming it has the same composition as LHS 6343 AB. 
There is a degeneracy between the inferred age of the system and the atmosphere of the brown dwarf (Figure 7). Specifically, a brown dwarf with the mass and radius of LHS $6343 \mathrm{C}$ would be expected to be significantly older if it were covered with optically thick clouds, as the clouds would keep the brown dwarf at a hotter internal adiabat. The models of Baraffe et al. (2003), which do not include clouds, suggest an age of approximately 5 Gyr, consistent with the cloudless models of Saumon \& Marley (2008). However, Saumon \& Marley (2008) predict a cloudy brown dwarf with a mass of LHS 6343 C and an age equal to the age of the universe would have a radius $2 \sigma$ larger than that observed for this object. This is consistent with the models of Burrows et al. (2011), who find the system must be very old if LHS $6343 \mathrm{C}$ has a thick layer of clouds. These authors claim thinner clouds or no clouds may be preferred by the data. Therefore, any additional observations which suggest the presence of clouds on LHS $6343 \mathrm{C}$ would be at odds with the predictions from theoretical brown dwarf model atmospheres.

The luminosity of LHS $6343 \mathrm{C}$ can be measured by observing its secondary eclipses as it passes behind LHS $6343 \mathrm{~A}$. In the $\mathrm{Ke}$ pler bandpass, we find the eclipse depth is $25 \pm 7 \mathrm{ppm}$. Between 1 and $3 \mu \mathrm{m}$, the depth is expected to be $0.1 \%$, observable with ground-based telescopes. In the $4.6 \mu \mathrm{m}$ Spitzer bandpass, the eclipse depth may be as large as $0.5 \%$ if the brown dwarf's atmosphere is cloud free. We will observe this system during four secondary eclipse events in Spitzer Cycle 10, observing two eclipses in each available IRAC bandpass. In addition to probing for extreme variability caused by patchy clouds in the atmosphere of LHS 6343 C, combining these observations with the Kepler secondary and ground-based $J H K$ photometry will enable us to measure a luminosity of this brown dwarf from the visible to the mid-IR. These observations will allow us to place the first data point on the brown dwarf mass-radius-metallicity-luminosity plane, testing the underconstrained brown dwarf atmospheric models in this parameter space for the first time.

We thank Luan Ghezzi and Jennifer Yee for helpful discussions that improved the quality of this manuscript.

The RV data presented herein were obtained at the W. M. Keck Observatory, which is operated as a scientific partnership among the California Institute of Technology, the University of California and the National Aeronautics and Space Administration. The Observatory was made possible by the generous financial support of the W. M. Keck Foundation. We are grateful to the entire Kepler team, past and present. Their tireless efforts were all essential to the tremendous success of the mission and the future successes of $\mathrm{K} 2$.

Some of the data presented in this paper were obtained from the Mikulski Archive for Space Telescopes (MAST). STScI is operated by the Association of Universities for Research in Astronomy, Inc., under NASA contract NAS5-26555. Support for MAST for non-HST data is provided by the NASA Office of Space Science via grant NNX13AC07G and by other grants and contracts. This paper includes data collected by the Kepler mission. Funding for the Kepler mission is provided by the NASA Science Mission directorate.

B.T.M. is supported by the National Science Foundation Graduate Research Fellowship under grant No. DGE1144469. J.A.J. is supported by generous grants from the David and Lucile Packard Foundation and the Alfred P. Sloan Foundation. C.B. acknowledges support from the Alfred P. Sloan Foundation.

The Robo-AO system is supported by collaborating partner institutions, the California Institute of Technology and the
Inter-University Centre for Astronomy and Astrophysics, and by the National Science Foundation under grant Nos. AST0906060, AST-0960343, and AST-1207891, by the Mount Cuba Astronomical Foundation, by a gift from Samuel Oschin.

We would like to thank the staff of both Palomar Observatory and the W. M. Keck Observatory for their support during our observing runs. Finally, we wish to acknowledge and recognize the very significant cultural role and reverence that the summit of Mauna Kea has always had within the indigenous Hawaiian community. We are most fortunate to have the ability to conduct observations from this mountain.

Facilities: Keck:I (HIRES), Kepler, Hale (TripleSpec), PO:1.5m (Robo-AO)

\section{APPENDIX}

\section{DERIVATION OF DIRECT MASS AND RADIUS MEASUREMENT}

Seager \& Mallén-Ornelas (2003) derive four directly observable parameters in an exoplanet light curve under a specific set of assumptions. Namely, they assume circular orbits, $M_{2} \ll M_{1}$, and that the third light contribution from a blended star is zero. None of these are true for the LHS 6343 system. As a result, the derivation which follows provides an analytic result which is exactly true when written in terms of physical parameters, but when common approximations for these parameters in terms of observables such as the transit duration, impact parameter, and relative flux decrement during transit are substituted for these parameters, the results below only approximate the truth. When calculating physical parameters using this method, care should be taken to avoid using these oversimplified expressions.

Following Seager \& Mallén-Ornelas (2003), the transit light curve enables a direct measurement of the stellar density $\rho_{\star}$ and the reduced semimajor axis and the stellar radius, $a / R_{\star}$. From these, the authors claim if the stellar mass-radius relation is known, then the stellar mass can be measured directly from the light curve. We show if the Doppler semiamplitude $K$ is known, the stellar mass can be measured exactly.

We know from Kepler's Third Law that, for two orbiting bodies with masses $M_{\star}$ and $m_{p}$ (by convention, $M_{\star}>m_{p}$ ) and orbital period $P$, that

$$
a=\left(\frac{G P^{2}\left(M_{\star}+m_{p}\right)}{4 \pi^{2}}\right)^{1 / 3},
$$

where $G$ is Newton's constant. The mean stellar density is defined for a star of mass $M_{\star}$ and radius $R_{\star}$ to be

$$
\rho_{\star}=\frac{3 M_{\star}}{4 \pi R_{\star}^{3}} .
$$

We can combine these two in such a way that we recover an expression for the mass ratio that depends only on observable parameters. We find

$$
1+\frac{m_{p}}{M_{\star}}=\left(\frac{3 \pi}{G P^{2}}\right)\left(\frac{1}{\rho_{\star}}\right)\left(\frac{a}{R_{\star}}\right)^{3} \equiv c_{1} .
$$

Famously, the Doppler semiamplitude $K$ observed in a RV survey is

$$
K=\left(\frac{2 \pi G}{P}\right)^{1 / 3} \frac{m_{p} \sin i}{\left(M_{\star}+m_{p}\right)^{2 / 3}} \frac{1}{\sqrt{1-e^{2}}} .
$$


Here, $i$ is the orbital inclination and $e$ the eccentricity, while all other variables retain their previous meaning. Rearranging this equation, we can once again write the mass ratio in terms of observable parameters only. In this case,

$$
\frac{m_{p}^{3}}{\left(M_{\star}+m_{p}\right)^{2}}=\frac{K^{3} P}{2 \pi G}\left(\frac{\sqrt{1-e^{2}}}{\sin i}\right)^{3} \equiv c_{2} .
$$

With two equations and two unknown masses, we can solve for the primary and secondary mass individually. We find

$$
\begin{aligned}
M_{\star} & =\frac{c_{1}^{2} c_{2}}{\left(c_{1}-1\right)^{3}} \\
& =\frac{\left(\frac{9 \pi}{2}\right)\left(\frac{1}{\rho_{\star}}\right)^{2}\left(\frac{a}{R_{\star}}\right)^{6}\left(\frac{K}{G P}\right)^{3}\left(\frac{\sqrt{1-e^{2}}}{\sin i}\right)^{3}}{\left[\left(\frac{3 \pi}{G P^{2}}\right)\left(\frac{1}{\rho_{\star}}\right)\left(\frac{a}{R_{\star}}\right)^{3}-1\right]^{3}}
\end{aligned}
$$

and

$$
\begin{aligned}
m_{p} & =\frac{c_{1}^{2} c_{2}}{\left(c_{1}-1\right)^{2}} \\
& =\frac{\left(\frac{9 \pi}{2}\right)\left(\frac{1}{\rho_{\star}}\right)^{2}\left(\frac{a}{R_{\star}}\right)^{6}\left(\frac{K}{G P}\right)^{3}\left(\frac{\sqrt{1-e^{2}}}{\sin i}\right)^{3}}{\left[\left(\frac{3 \pi}{G P^{2}}\right)\left(\frac{1}{\rho_{\star}}\right)\left(\frac{a}{R_{\star}}\right)^{3}-1\right]^{2}} .
\end{aligned}
$$

From the stellar density, the calculated mass can be used to measure the stellar radius. Plugging this equality in to Equation (A2) above, we find that

$$
R_{\star}=\frac{\left(\frac{3}{2}\right)\left(\frac{1}{\rho_{\star}}\right)\left(\frac{a}{R_{\star}}\right)^{2}\left(\frac{K}{G P}\right)\left(\frac{\sqrt{1-e^{2}}}{\sin i}\right)}{\left[\left(\frac{3 \pi}{G P^{2}}\right)\left(\frac{1}{\rho_{\star}}\right)\left(\frac{a}{R_{\star}}\right)^{3}-1\right]} .
$$

From a known stellar radius, the transit depth can be used to measure the planet radius directly. For a flux decrement $\Delta F$,

$$
R_{p}=R_{\star} \sqrt{\Delta F} .
$$

Therefore, by measuring the stellar density, reduced semimajor axis, orbital period, transit depth, inclination, eccentricity, and Doppler semiamplitude, we can measure the stellar and planetary mass and radius. Moreover, since the companion is transiting, we know $\sin i \approx 1$.

Dawson \& Johnson (2012) present equations for the physical parameters above in terms of parameters directly observable from the light curve. Specifically, they find, in the limit of $m_{p} \ll M_{\star}$,

$$
\frac{a}{R_{\star}}=\frac{2 \delta^{1 / 4} P}{\pi \sqrt{T_{14}^{2}-T_{23}^{2}}} \frac{\sqrt{1-e^{2}}}{1+e \sin w}
$$

and

$$
\rho_{\star}=\left[\frac{2 \delta^{1 / 4}}{\sqrt{T_{14}^{2}-T_{23}^{2}}}\right]^{3}\left(\frac{3 P}{G \pi^{2}}\right)\left(\frac{\sqrt{1-e^{2}}}{(1+e \sin w)}\right)^{3} .
$$

Here, $\delta=\left(R_{p} / R_{\star}\right)^{2}$ is the fractional transit depth, or the relative areas of the transiting companion and the host star. $T_{14}$ is the transit duration from first to fourth contact (including ingress

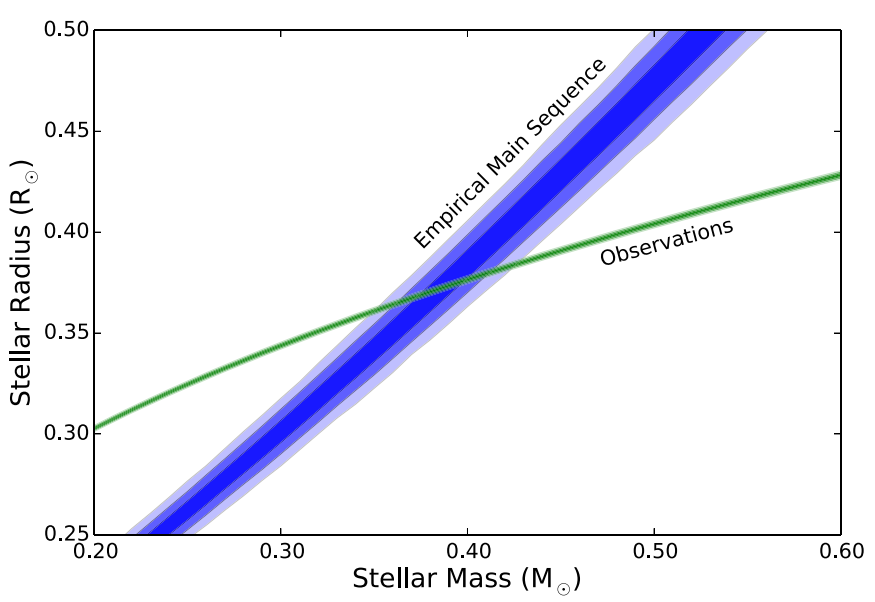

Figure 8. Mass-radius relation for LHS 6343 A from the observed transit light curve and RV observations (green), plotted with the mass-radius relation for $\mathrm{K}$ and M dwarfs (blue) of Boyajian et al. (2012). There are many possible stellar masses and radii which are formally allowed, but are unphysical. By combining weak constraints from empirical observations of the main sequence, a robust direct measurement on the mass and radius of both LHS 6343 A and LHS 6343 C can be made.

and egress), and $T_{23}$ is the transit duration from second to third contact (excluding ingress and egress).

If we substitute these into our above equations for the stellar mass and radius, we find our expressions for the mass and radius are undefined. Specifically, our denominator, $c_{1}-1$ is undefined at $m=0$. Our equations above work specifically in the case where the mass of the companion is not negligible. This is because the stellar density cannot be measured exactly from the light curve alone. While often neglected in exoplanet studies, the true observable is $\left(M_{\star}+m_{p}\right) / R_{\star}^{3}$. In cases where the mass ratio is large, this value approaches $M_{\star} / R_{\star}^{3}$, enabling the stellar density to be approximated well. For the case of a Jupiter-sized planet transiting a Sun-like star, such an approximation is reasonable. However, this approximation breaks down for small mass ratios. In this case, an additional constraint is required.

An additional constraint can be provided by using the mass ratio, which can be measured by observing ellipsoidal variations in the full phase curve (Loeb \& Gaudi 2003). Ellipsoidal variations have been used both to confirm transiting planets (e.g., Mislis \& Hodgkin 2012) and to measure the mass ratios of already-confirmed planets (e.g., Welsh et al. 2010; Jackson et al. 2012). By including such an observation, the degeneracy between the stellar density and mass ratio can be broken and the stellar mass measured directly.

When both the mass ratio is small and ellipsoidal variations cannot be observed from the light curve, the masses can still be measured directly if the star can be assumed to fall on the main sequence, as outlined by Seager \& Mallén-Ornelas (2003). For a fixed transit depth, reduced semimajor axis, and Doppler semiamplitude, a star's inferred mass is related to the star's predicted radius such that $M \propto R^{>3}$, with the exact coefficient depending on the host-companion mass ratio (and approaching three as the mass ratio becomes infinite). Since the stellar main sequence has a significantly different mass-radius relation, this information can be used to rule out many unphysical transit models. An example of this is shown as Figure 8.

Because a nonzero mass ratio is required, this method is likely only applicable when the companion is a hot Jupiter, transiting brown dwarf, or low-mass stellar companion. Moreover, it requires precise knowledge of both the Doppler semiamplitude 
and transit parameters. Therefore, the potential of this method is likely limited at present to hot transiting companions orbiting bright host stars. Yet for these cases this technique may be very useful, especially when stellar evolutionary models may have systematic errors, such as when the host is an M dwarf or subgiant star.

\section{REFERENCES}

Allard, F., \& Freytag, B. 2010, HiA, 15, 756

Baraffe, I., Chabrier, G., Barman, T. S., Allard, F., \& Hauschildt, P. H. 2003, A\&A, 402, 701

Baranec, C., Riddle, R., Law, N. M., et al. 2014, ApJL, 790, L8

Bastien, F. A., Stassun, K. G., Basri, G., \& Pepper, J. 2013, Natur, 500, 427

Borucki, W. J., Koch, D., Basri, G., et al. 2010, Sci, 327, 977

Bouchy, F., Bonomo, A. S., Santerne, A., et al. 2011a, A\&A, 533, A83

Bouchy, F., Deleuil, M., Guillot, T., et al. 2011b, A\&A, 525, A68

Boyajian, T. S., von Braun, K., van Belle, G., et al. 2012, ApJ, 757, 112

Burgasser, A. J., Gillon, M., Faherty, J. K., et al. 2014, ApJ, 785, 48

Burrows, A., Heng, K., \& Nampaisarn, T. 2011, ApJ, 736, 47

Burrows, A., Hubbard, W. B., Lunine, J. I., \& Liebert, J. 2001, RvMPh, 73, 719

Claret, A., \& Bloemen, S. 2011, A\&A, 529, A75

Covey, K. R., Lada, C. J., Román-Zúñiga, C., et al. 2010, ApJ, 722, 971

Crepp, J. R., Johnson, J. A., Fischer, D. A., et al. 2012, ApJ, 751, 97

Cushing, M. C., Rayner, J. T., \& Vacca, W. D. 2005, ApJ, 623, 1115

Cushing, M. C., Vacca, W. D., \& Rayner, J. T. 2004, PASP, 116, 362

Dawson, R. I., \& Johnson, J. A. 2012, ApJ, 756, 122

Deleuil, M., Deeg, H. J., Alonso, R., et al. 2008, A\&A, 491, 889

Díaz, R. F., Damiani, C., Deleuil, M., et al. 2013, A\&A, 551, L9

Díaz, R. F., Montagnier, G., Leconte, J., et al. 2014, A\&A, 572, A109

Dotter, A., Chaboyer, B., Jevremović, D., et al. 2008, ApJS, 178, 89

Dupuy, T. J., Liu, M. C., \& Ireland, M. J. 2009, ApJ, 692, 729

Dupuy, T. J., Liu, M. C., \& Ireland, M. J. 2014, ApJ, 790, 133

Faherty, J. K., Beletsky, Y., Burgasser, A. J., et al. 2014, ApJ, 790, 90

Foreman-Mackey, D., Hogg, D. W., Lang, D., \& Goodman, J. 2013, PASP, 125,306

Gilliland, R. L., Brown, T. M., Christensen-Dalsgaard, J., et al. 2010, PASP, 122,131

Girardi, L., Bertelli, G., Bressan, A., et al. 2002, A\&A, 391, 195

Goodman, J., \& Weare, J. 2010, Commun. Appl. Mathe. Comput. Sci., 5, 65

Herrero, E., Lanza, A. F., Ribas, I., Jordi, C., \& Morales, J. C. 2013, A\&A, 553, A66
Herrero, E., Lanza, A. F., Ribas, I., et al. 2014, A\&A, 563, A104

Herter, T. L., Henderson, C. P., Wilson, J. C., et al. 2008, Proc. SPIE, 7014, 70140X

Howard, A. W., Johnson, J. A., Marcy, G. W., et al. 2010, ApJ, 721, 1467

Huber, D., Carter, J. A., Barbieri, M., et al. 2013, Sci, 342, 331

Irwin, J., Buchhave, L., Berta, Z. K., et al. 2010, ApJ, 718, 1353

Jackson, B. K., Lewis, N. K., Barnes, J. W., et al. 2012, ApJ, 751, 112

Jenkins, J. M., Caldwell, D. A., Chandrasekaran, H., et al. 2010, ApJL, 713, L87

Johnson, J. A., Apps, K., Gazak, J. Z., et al. 2011, ApJ, 730, 79

Knutson, H. A., Fulton, B. J., Montet, B. T., et al. 2014, ApJ, 785, 126

Latham, D. W., Stefanik, R. P., Mazeh, T., Mayor, M., \& Burki, G. 1989, Natur, 339,38

Law, N. M., Hodgkin, S. T., \& Mackay, C. D. 2006, MNRAS, 368, 1917

Law, N. M., Morton, T., Baranec, C., et al. 2014, ApJ, 791, 35

Littlefair, S. P., Casewell, S. L., Parsons, S. G., et al. 2014, MNRAS, 445, 2106

Liu, M. C., Fischer, D. A., Graham, J. R., et al. 2002, ApJ, 571, 519

Loeb, A., \& Gaudi, B. S. 2003, ApJL, 588, L117

Mandel, K., \& Agol, E. 2002, ApJL, 580, L171

Mayor, M., \& Queloz, D. 1995, Natur, 378, 355

Mislis, D., \& Hodgkin, S. 2012, MNRAS, 422, 1512

Moutou, C., Bonomo, A. S., Bruno, G., et al. 2013, A\&A, 558, L6

Muirhead, P. S., Becker, J., Feiden, G. A., et al. 2014, ApJS, 213, 5

Nakajima, T., Oppenheimer, B. R., Kulkarni, S. R., et al. 1995, Natur, 378, 463

Oshagh, M., Boué, G., Haghighipour, N., et al. 2012, A\&A, 540, A62

Patel, S. G., Vogt, S. S., Marcy, G. W., et al. 2007, ApJ, 665, 744

Pineda, J. S., Bottom, M., \& Johnson, J. A. 2013, ApJ, 767, 28

Rayner, J. T., Cushing, M. C., \& Vacca, W. D. 2009, ApJS, 185, 289

Rebolo, R., Zapatero Osorio, M. R., \& Martín, E. L. 1995, Natur, 377, 129

Rojas-Ayala, B., Covey, K. R., Muirhead, P. S., \& Lloyd, J. P. 2012, ApJ, 748,93

Saumon, D., \& Marley, M. S. 2008, ApJ, 689, 1327

Seager, S., \& Mallén-Ornelas, G. 2003, ApJ, 585, 1038

Siverd, R. J., Beatty, T. G., Pepper, J., et al. 2012, ApJ, 761, 123

Southworth, J. 2011, MNRAS, 417, 2166

Stassun, K. G., Mathieu, R. D., \& Valenti, J. A. 2006, Natur, 440, 311

Torres, G. 1999, PASP, 111, 169

Triaud, A. H. M. J., Hebb, L., Anderson, D. R., et al. 2013, A\&A, 549, A18

Welsh, W. F., Orosz, J. A., Seager, S., et al. 2010, ApJL, 713, L145

Wilson, J. C., Henderson, C. P., Herter, T. L., et al. 2004, Proc. SPIE, 5492,1295

Winget, D. E., Nather, R. E., Clemens, J. C., et al. 1991, ApJ, 378, 326

York, D. G., Adelman, J., Anderson, J. E., Jr, et al. 2000, AJ, 120, 1579 